\title{
Opportunistic Hybrid
}

Communications Systems for

Distributed PV Coordination

\section{Bri-Mathias Hodge}

National Renewable Energy Laboratory 


\section{Project Innovation and Objective}

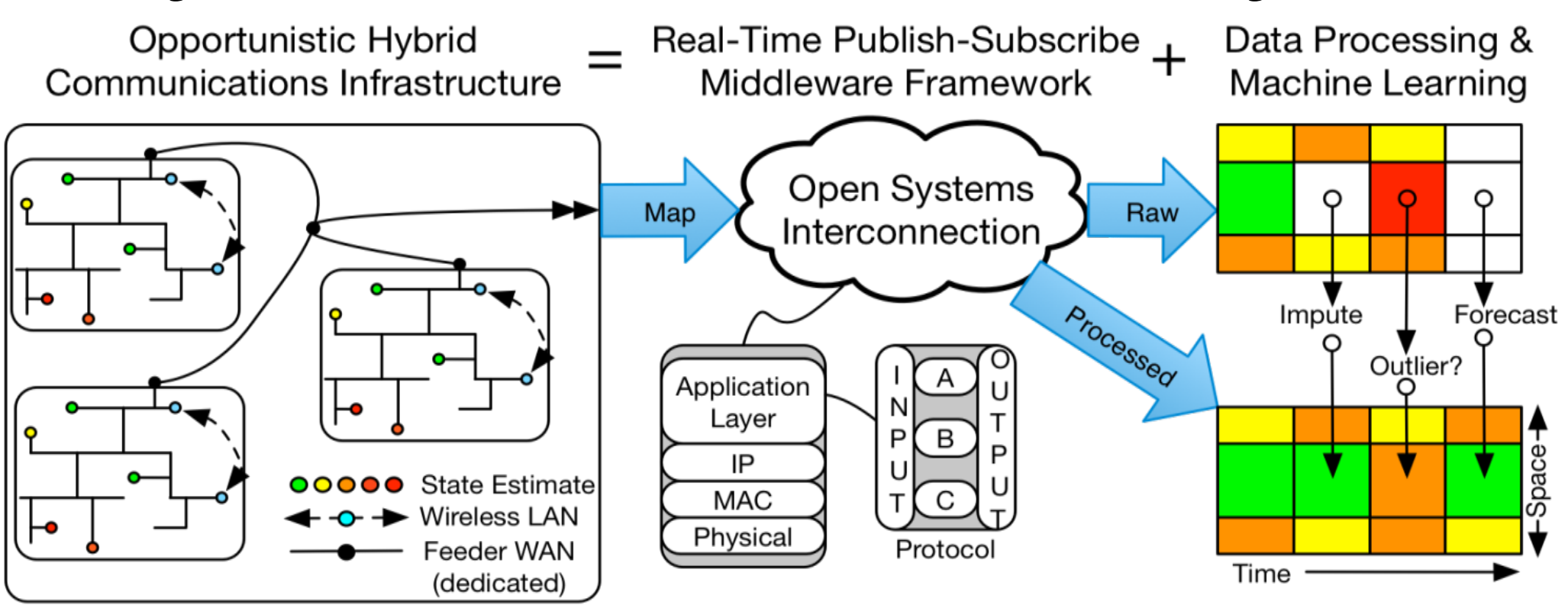

Innovation: To highly use the diverse communication infrastructure existed in the power system, we aim to develop the hybrid communication system at the least cost to coordinate distributed PV generation.

Objective: Communicate the state of the grid from the inverter to the system operator by fullscale, operational implementation of the opportunistic hybrid communication system:

- Hybrid: various communications pathways, e.g. LoWPAN, PLC, WiFi, WiMAX etc.

- Opportunistic: route messages through each of these systems based on recent data about latency and availability to ensure reliable message passing. 


\section{The Smart Grid Communication}

LEGEND

(( LoWPAN (e.g., ZigBee, IEEE 802.15.4)

Wireless LAN (e.g., IEEE 802.11)

I NSP Broadband Wireless (e.g., LTE, IEEE 802.16)

PLC (e.g., Broad band (IEEE 1901, HomePlug); Narrow band (IEEE 1901.2))

Ethernet (e.g., IEEE 802.3)

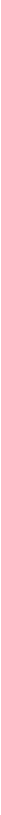

Neighborhood Area Network
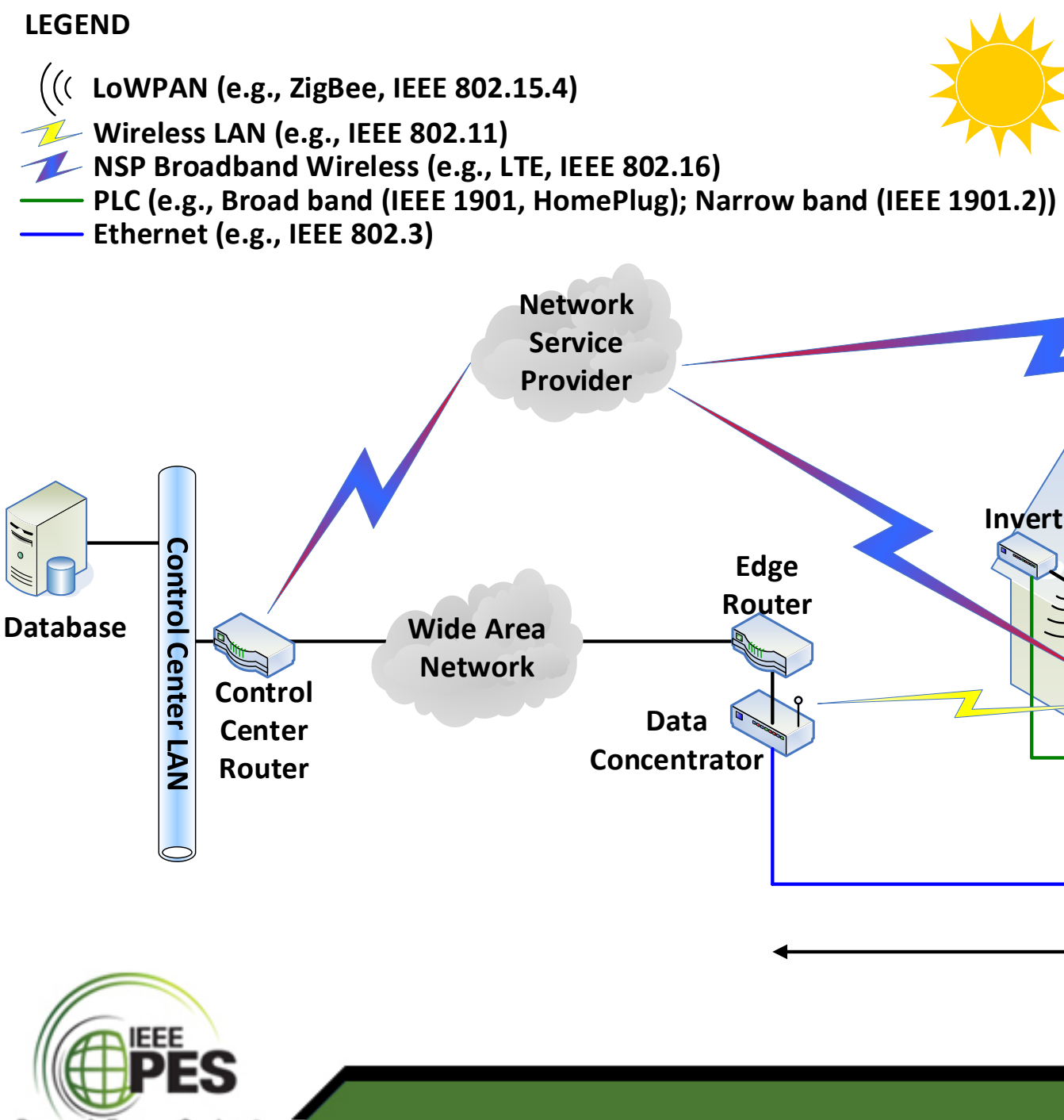

Home Area Network 


\section{Potential Use Cases and Applications}

- Three design metrics: Latency, Throughput, and Packet Loss Rate for different hybrid communication architecture designs;

- Monitoring Functions: Distributed state estimation algorithms for distributed PV systems and distribution power systems;

- Control Functions: Distributed PV dispatch commands from DSO or TSO;

- Ancillary Services from utility point of view. 


\section{Hybrid Communication Design Stages}

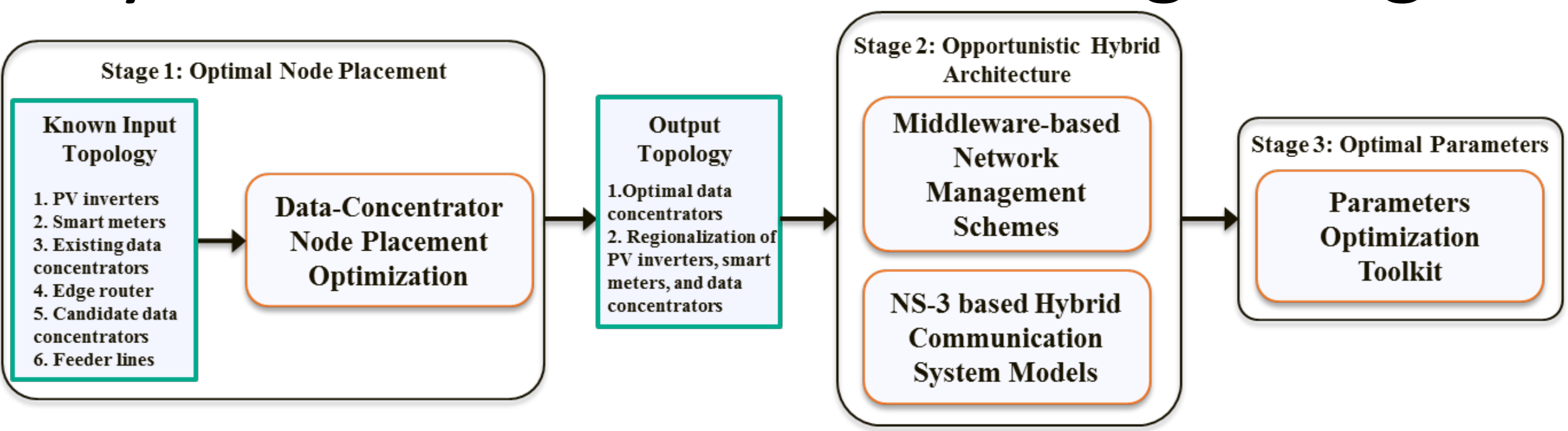

Reference Test Case A (RTC-A)

- Taxonomy feeder R2-25.00-1 from DOE's Modern Grid Initiative representing moderate urban environment

- System of 1080 nodes on a distribution feeder 


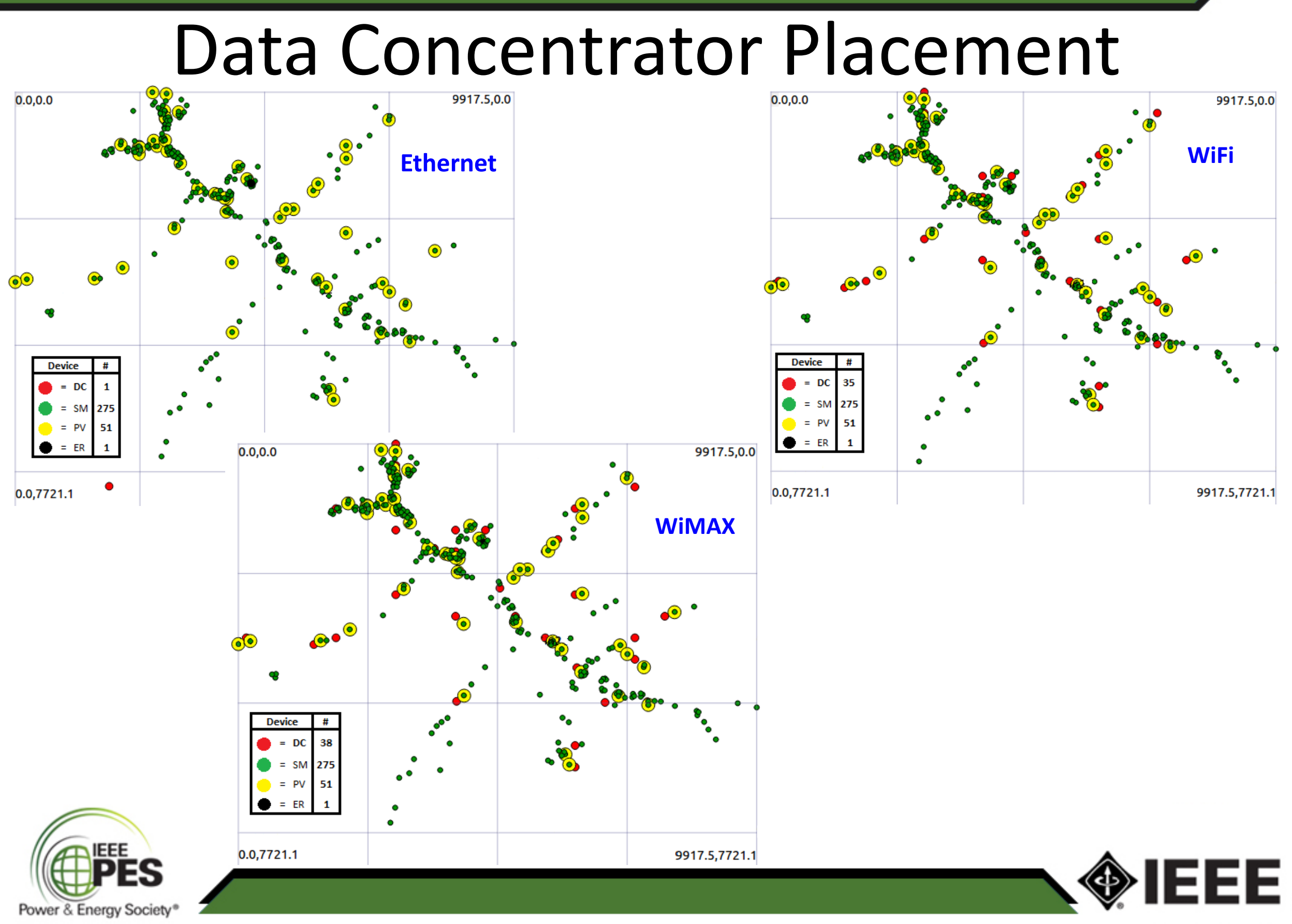




\section{Opportunistic Hybrid Communication Models}

PV Inverter

\section{\begin{tabular}{|c|}
\hline \multicolumn{2}{|c|}{ Application } \\
\hline Middleware
\end{tabular}}
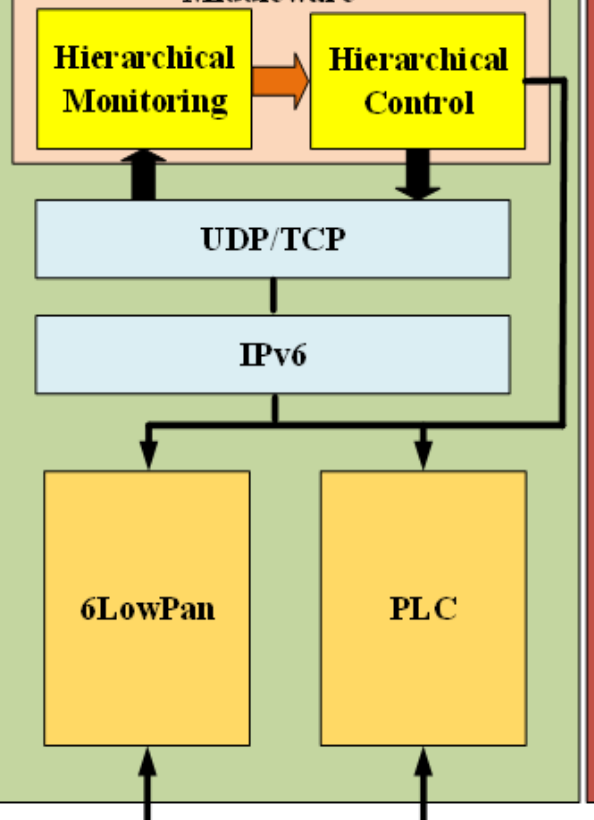

Sinart Meter

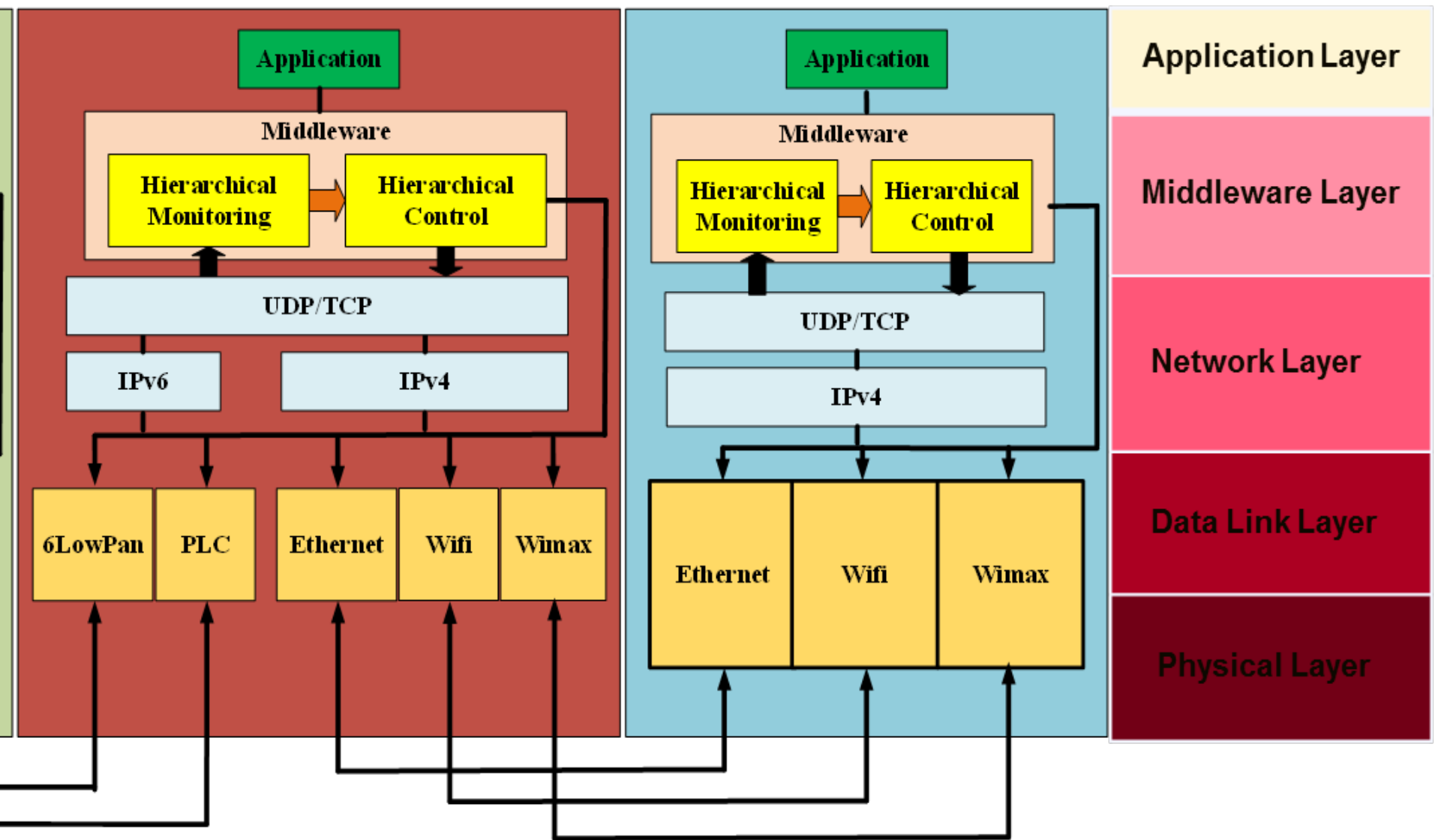

Concentrator

Home Area Network (HAN): BPLC, NPLC, LoWPAN

Neighborhood Area Network (NAN): Ethernet, WiFi, WiMAX Total 9 hybrid designs

Data 


\section{Optimal Performance and Parameters}

\section{Variables: Date rate and Packet size}

TABLE IV: Optimal Parameter Results for Nine Hybrid Designs

\begin{tabular}{|c|c|c|c|c|c|}
\hline Hybrid Type & Data Rate (Kbps) & Packet Size (Bytes) & Latency (ms) & Throughput (Kbps) & Packet Loss Rate (\%)) \\
\hline LoWPAN-Ethernet & 56 & 631 & 5.144 & 56.003 & 0 \\
\hline LoWPAN-WiFi & 55.98 & 251 & 5.622 & 55.981 & 0.033 \\
\hline LoWPAN-WiMAX & 55.85 & 795 & 11.75 & 55.849 & 0 \\
\hline BPLC-Ethernet & 55.804 & 699 & 7.098 & 55.819 & 0 \\
\hline BPLC-WiFi & 55.043 & 588 & 11.436 & 54.875 & 0.622 \\
\hline BPLC-WiMAX & 55.551 & 70 & 7.102 & 55.559 & 0 \\
\hline NPLC-Ethernet & 55.987 & 643 & 26.922 & 56.011 & 0.021 \\
\hline NPLC-WiFi & 55.862 & 635 & 33.256 & 55.676 & 0.497 \\
\hline NPLC-WiMAX & 56 & 756 & 41 & 56.032 & 0 \\
\hline
\end{tabular}




\section{Distributed State Estimation Techniques}
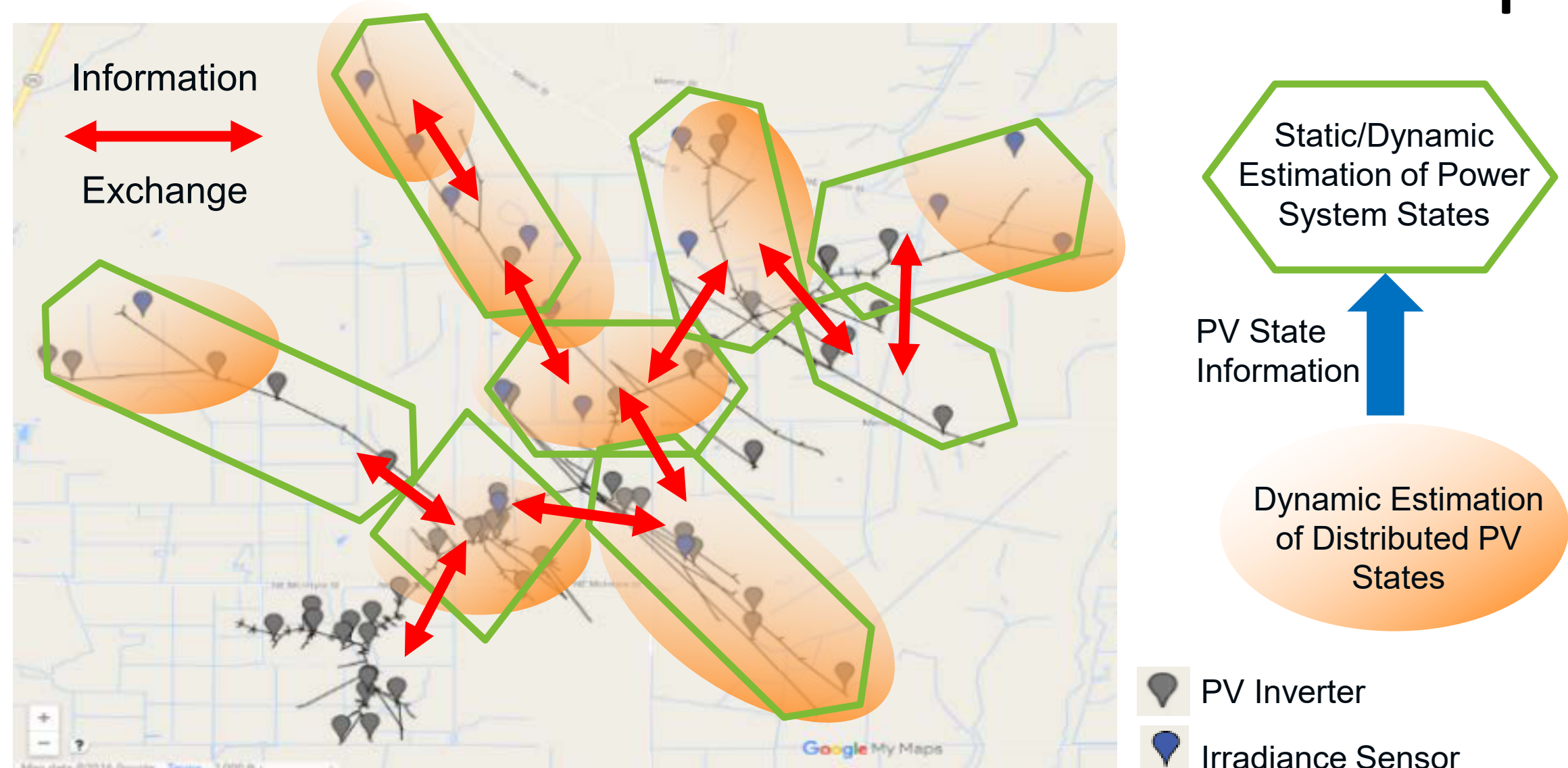

\section{Dynamic Estimation of Distributed PV States}

PV Inverter

$P$ Irradiance Sensor

- Improve PV generation efficiency by using dynamic estimation of distributed PV states

- Enable monitoring and control of distribution PV generation using distribution state

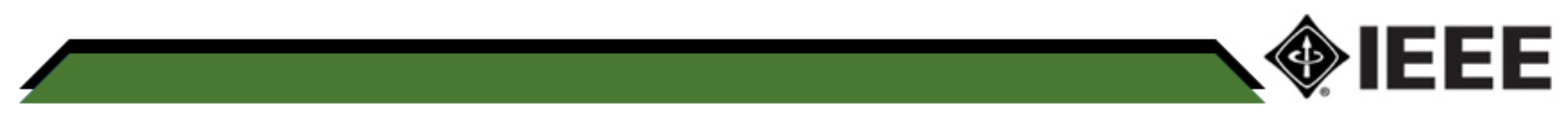




\section{Distributed PV System State Estimation}

$>$ Multi Rate and Event DRIven Kalman Kriging (MREDRIKK) Filter [2].
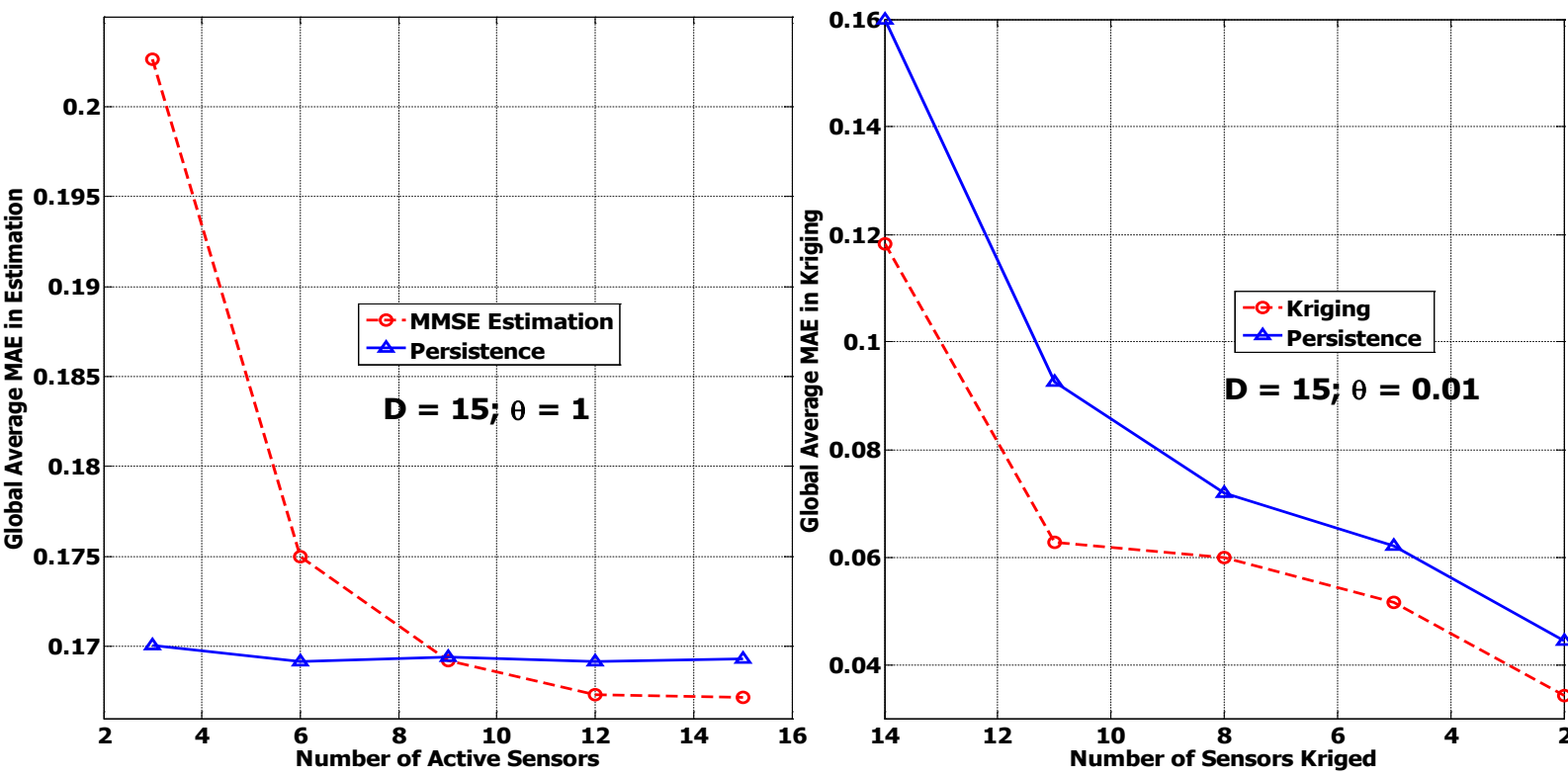

Estimation and Kriging Performs Better Than Persistence.

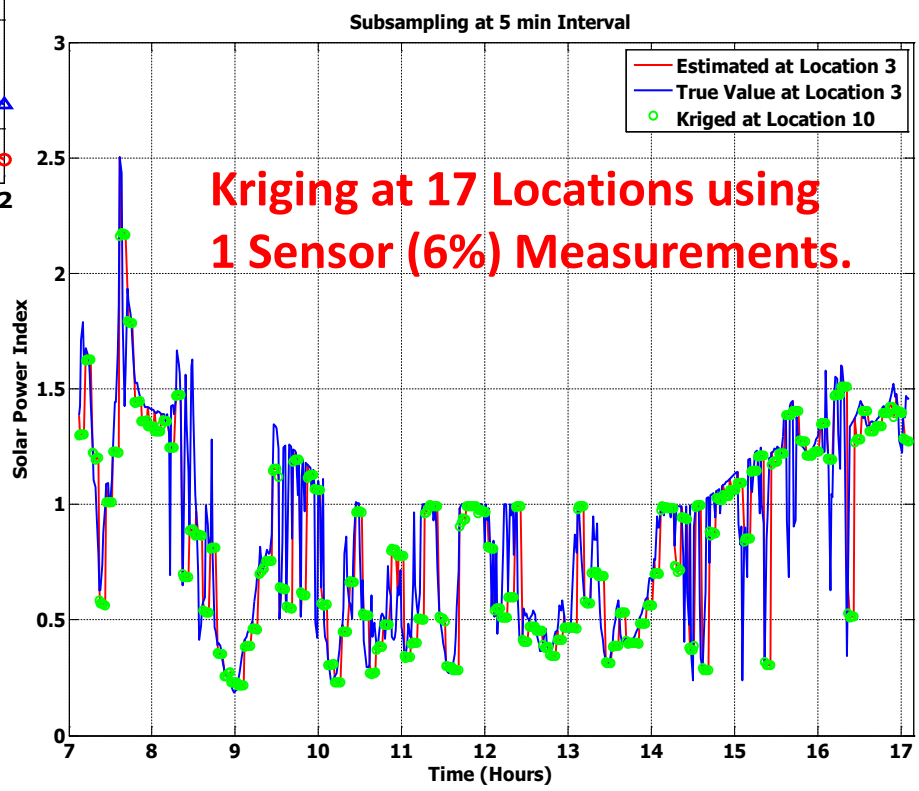




\section{Distributed State Estimation for Power Distribution System}

\section{$>$ Spectral clustering based automatic regionalization [3].}

\section{$>$ Distributed Ladder Iterative State Estimation (DiLISE) [4].}

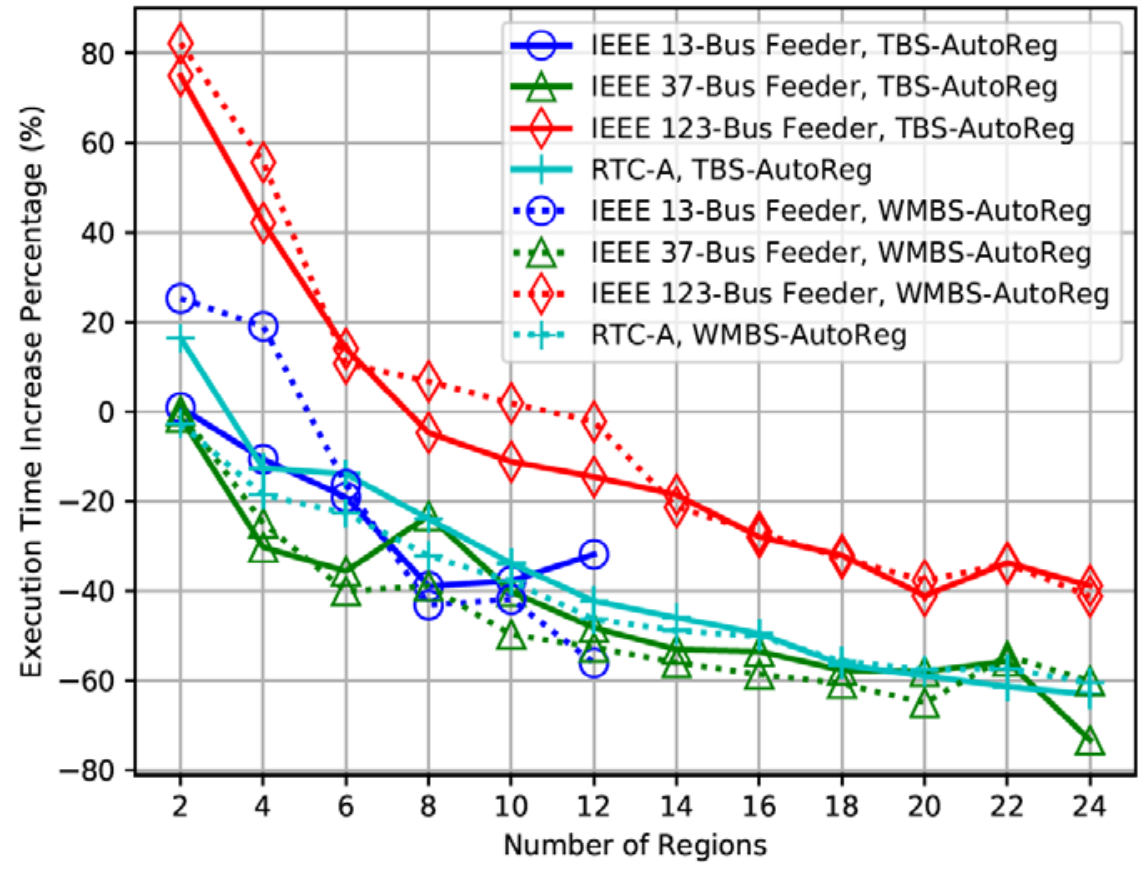

Performance of DiLISE for Different Test Cases

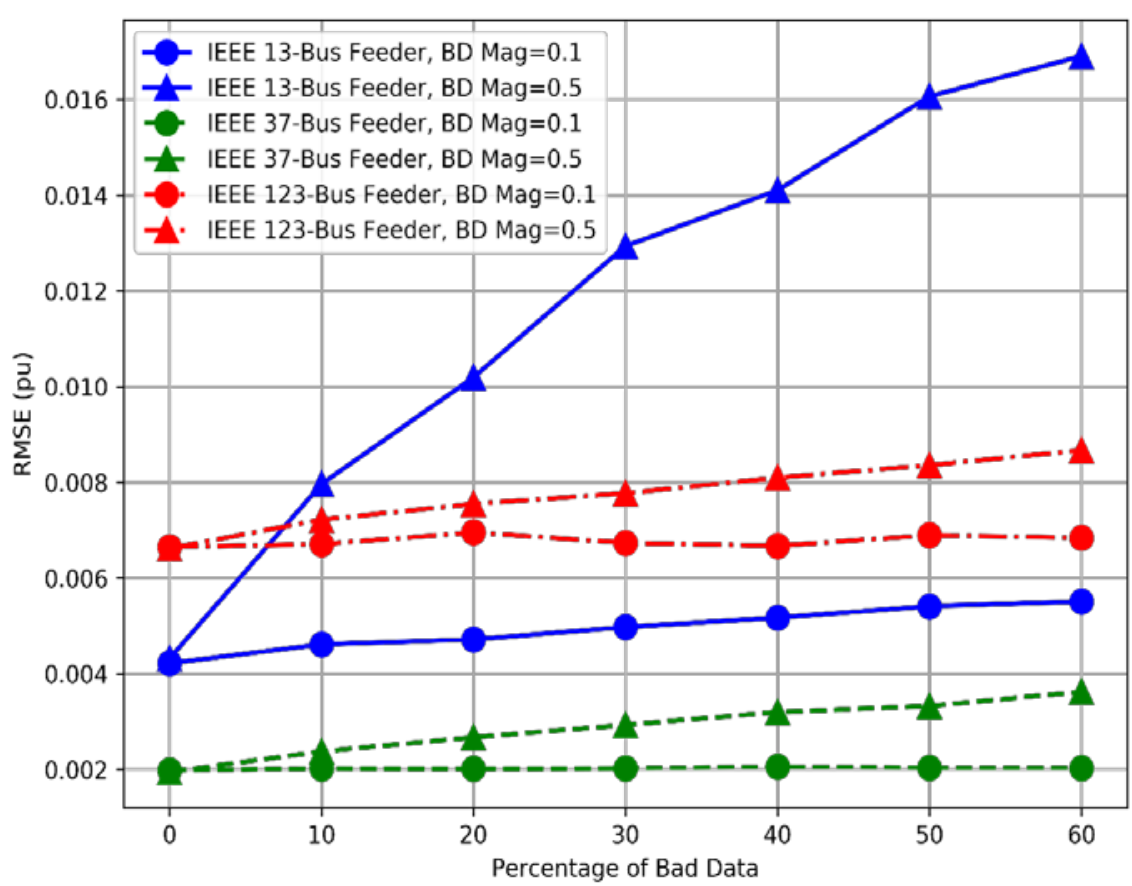

Impact of Bad Data on DiLISE Algorithm

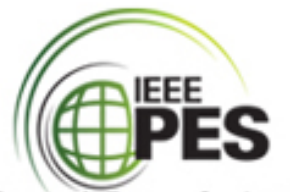




\section{Communication and Power HIL Test}

Power System Model (Feeder RS-25.00-1)

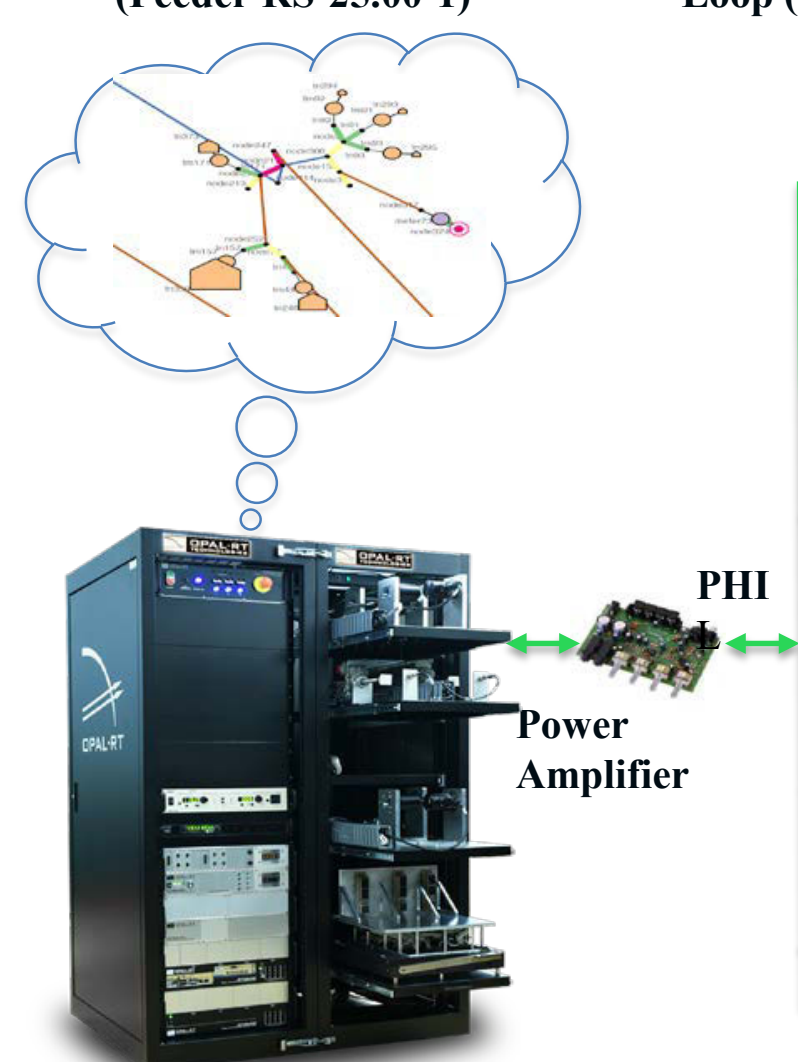

Power Hardware in the

Loop (PHIL)

f--- 6LoWPAN---

Communication Hardware

in the Loop (CHIL)

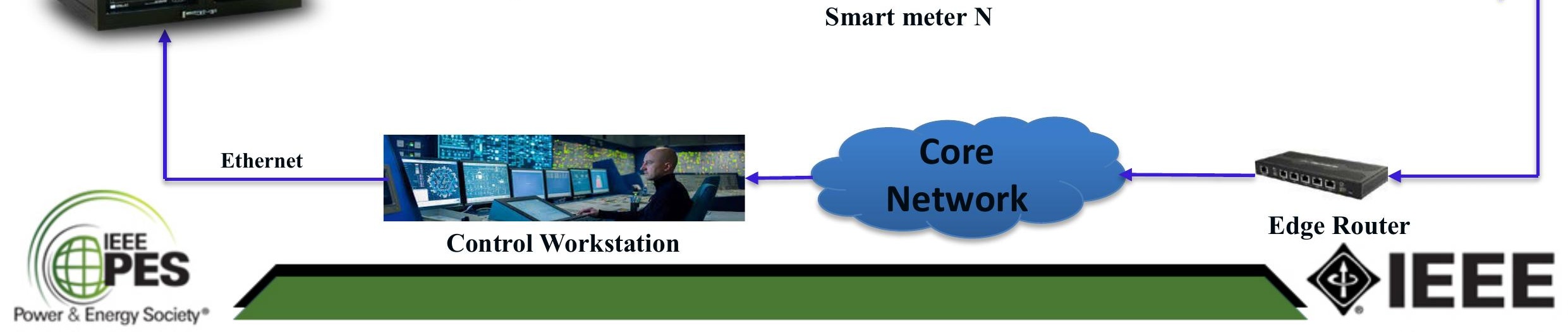




\section{In Progress...}

- Integration of NS-3 simulation testbed with communication and power hardware-in-the-loop (HIL) testbed.

- Integration of distributed state estimation algorithms for power distribution system and distributed PV systems.

\section{Reference}

[1] Zhang et al., "Hybrid Communication Architectures for Distributed Smart Grid Applications", $1^{\text {st }}$ revision submitted to MDPI Energies. [2] Alam et al., "Multi-Rate and Event-DRIven Kalman Kriging Filter for Distributed PV System State Estimation," $1^{\text {st }}$ revision submitted to IEEE Trans. Smart Grid.

[3] Wang et al., "Automatic Regionalization Algorithm for Distributed State Estimation in Power Systems", IEEE GlobalSIP 2016.

[4] Wang et al., "Distributed Ladder Iterative State Estimation and Automatic Regionalization for Distribution Systems", $1^{\text {st }}$ revision submitted to IEEE Trans. Smart Grid. 


\section{Thank You!!}

\section{Bri.Mathias.Hodge@nrel.gov}

